\author{
Asian Journal of \\ Medical and Biological Research \\ ISSN 2411-4472 (Print) 2412-5571 (Online) \\ www.ebupress.com/journal/ajmbr
}

\title{
Article \\ Prevalence of etiologic agents causing invasive bacterial disease and evaluation of their antibiotic susceptibility pattern
}

\author{
Md. Mijanur Rahman ${ }^{1 *}$, Mohammad Sharif Uddin ${ }^{2}$, Salma Aktar ${ }^{1}$, Mohammad Shaokat Ali $^{3}$, Partha Paul ${ }^{1}$, Md. \\ Mahmudul Hasan ${ }^{1}$ and Shah Md. Ataur Rahman ${ }^{1}$ \\ ${ }^{1}$ Department of Microbiology, Noakhali Science and Technology University, Noakhali-3814, Bangladesh \\ ${ }^{2}$ Department of Microbiology, University of Chittagong, Chittagong-4331, Bangladesh \\ ${ }^{3}$ Department of Applied Chemistry and Chemical Technology, Faculty of Food Science and Technology, \\ Chittagong Veterinary and Animal Sciences University, Khulshi, Chittagong-4225, Bangladesh
}

*Corresponding author: Md. Mijanur Rahman, Lecturer, Department of Microbiology, Noakhali Science \& Technology University (NSTU), Noakhali-3814, Bangladesh. Phone: +8801823103818; E-mail: mijan.cu91@gmail.com

Received: 07 October 2018/Accepted: 30 October 2018/ Published: 30 December 2018

\begin{abstract}
Invasive bacterial diseases (IBD) cause significant morbidity and mortality which leads to enormous health suffering and economic frustration in Bangladesh especially in people with resource poor region. The prevalence of IBD may vary even in different regions in a country. To understand the prevalence of etiologic agents causing IBD and determining their response to commonly used standard antibiotics we conducted a retrospective cross sectional study in rural town of Bangladesh on patient attended for blood culture with IBD sign-symptoms in well reputed microbiology lab. We considered IBD patients (118) of all age groups (0-85 years) both gender (male 54\% and female 46\%). 25\% (30) blood culture of all IBD patients found bacterial growth positive including contaminant Staphylococcus sp. (10). The isolated IBD etiologic agents are S. aureus (10), Salmonella sp. (5), Salmonella Paratyphi (1), E. coli (2), Shigella sp. (1), Pseudomonas sp. (1). We could have retrieved antibiotic susceptibility testing data of five isolates including S. aureus (1), Salmonella sp. (2), Salmonella Paratyphi (1), Pseudomonas sp. (1); which showed except Salmonella sp. all isolates are sensitive to tested commonly used standard antibiotics. Two non typhoidal Salmonella sp. showed intermediate sensitivity to ciprofloxacin $(5 \mu \mathrm{g})$ which indicate reconsideration of choosing non typhoidal Salomonella sp. infection with ciprofloxacin $(5 \mu \mathrm{g})$. As it is a retrospective, not well organized cross sectional study; these findings may not represent the entire actual scenario of IBD in the region. As a consequence, a well organizing and adequately powered study must need to be conducted.
\end{abstract}

Keywords: invasive bacterial diseases; non typhoidal Salmonella sp.; antibiotic susceptibility

\section{Introduction}

Globally invasive bacterial diseases (IBD) are major cause of morbidity and mortality. In developing countries like Bangladesh IBD in children under 5 years is the significant public health problem. Invasive bacterial infection can be defined as the detection of bacteria in the normally sterile body fluids such as blood, cerebrospinal fluid (CSF), joint fluid, pleural fluid, pericardial fluid, bone aspirate, or a deep tissue abscess (Schuchat et al., 2001). Streptococcus pneumoniae, Haemophilus influenzae, Neisseria meningitidis, Streptococcus agalactiae, Streptococcus pyogenes, Staphylococcus aureus, Salmonella species, Listeria species, and Escherichia coli are major bacteria involved in invasive infections in children especially in immunocompetent children (Nam and Lee, 1998; Lee et al., 2005). Most of the complications and mortality caused due to pneumonia, sepsis (Shann, 1995; Mullholland, 1999) and meningitis (Greenwood, 1987; Murray and Lopez, 1996). Pneumonia is the leading cause of mortality among the children under 5 years which globally 
account to $19 \%$ per year (Jennifer et al., 2005). Streptococcus pneumoniae and Haemophilus influenzae type-b are mainly involved in more than $50 \%$ and $20 \%$ of severe pneumonia cases with high mortality rate respectively (UNICEF/WHO, 2006).

Para-typhoid and typhoid fever are also the leading cause of child death (Denny and, Loda, 1886; Shann, 1986). Typhoid fever remains substantial public health concern in developing countries account to 16 million cases with 600,000 related deaths worldwide (Hossain et al., 2000; Yichun, 2003).

Emerging of multiple drug resistance bacteria is a significant public health concern around the globe. In the case of invasive non-typhoidal Salmonella (iNTS) ciprofloxacin is recommended as first-line treatment and levofloxacin, moxifloxacin, cotrimoxazole, or the broad spectrum cephalosporins, ceftrioxone and cefotaxime are used as alternatives. Multidrug resistant NTS are involved in increased morbidity and are important health concern in both animals and humans. (Kariuki and Dougan, 2014; Guerrant et al., 1990).

Because of lacking empirical data and evidence based intervention the use of the antibiotics has been increasing rapidly all over the world. Klein et al. (2018) have conducted a new global study which aimed to determine trends in antibiotics use from 2010-2015 in 76 countries. They compared total consumption of antibiotics between low-middle income countries (LMIC) e.g., India and China, and high-income countries (HIC) such as the UK and US. According to this study, between 2000-2015, global antibiotic consumption increased by $65 \%$ from 21.1 to 34.8 billion defined daily doses (DDDs), e.g. a single antibiotic capsule or injection - of antibiotics to 34.8 billion DDDs. The antibiotic consumption rate increased by $39 \%$ from 11.3 to 15.7 DDDs per 1,000 inhabitants per day over the 15 years of study periods. Increased consumption of antibiotics in the low- middle income countries was the primary reason for the increase in the global consumption. In low-middle countries antibiotic consumption raises $114 \%$ (11.4 to 24.5 billion DDDs) and the consumption rate increased $77 \%$ ( 7.6 to 13.5 DDDs per 1,000 inhabitants per day). Among LMICs India, China and Pakistan were the highest antibiotic consumers in 2015. In high-income countries (HICs) the total consumption of antibiotic increased by $6.6 \%(9.7$ to 10.3 billion DDDs) and the rate of consumption increased by 4\% (26.8 to 25.7 DDDs per 1,000 inhabitants per day). In 2015 US, France and Italy were the highest antibiotic consumer among HICs (Klein et al., 2018). Over use or injudicious use of antibiotics may contribute in emerging of multi-drug resistant bacterial strain.

In this retrospective cross sectional study, we aimed to assess the prevalence of invasive bacterial disease causing etiologic agents and their antibiotic susceptibility pattern in a rural town in Bangladesh from January to September 2018.

\section{Materials and Methods}

\subsection{Patient criteria}

We retrospectively collected the data of 118 patients who meets WHO criteria of invasive bacterial disease (IBD) and performed blood culture in a reputed microbiology laboratory in Bangladesh.

\subsection{Blood collection and processing}

BD Bactec-120 system has been used for blood culture along with combination of conventional plate culture method. 1-3 ml of blood were taken in Bactec PD Plus Aerobic Blood Culture bottle and inserted into Bactec120 machine, and incubated at $35.5^{\circ} \mathrm{C}$ for 5 days. Bactec fluorescent series instrument has made significant impact on laboratory blood culture practice by enhancing sensitivity and reducing time to detect numerous pathogens in blood. This instrument swiftly detects presence of microorganisms in Bactec Ped Plus/F culture vials (containing Soyabean Casein Digest Broth and other necessary ingredients) and produces a positive signal based on detecting $\mathrm{CO}_{2}$ in growth media. After getting positive signal from the machine, it was suspected that there might presence some organisms or pathogens, then the positive culture bottle was taken out from the machine and sub-cultured on to three media plates such as Blood agar, Chocolate agar and MacConkey agar. The organisms are then finally identified on the basis of their morphological characteristics including size and shape of the organism, arrangement of the cells, presence or absence of the spores, regular or irregular forms, acid fastness, gram reaction etc.; cultural and physiological characteristics including $\mathrm{H}_{2} \mathrm{~S}$ production, nitrate reduction, deep glucose agar test, fermentation of different carbohydrates etc. All these characteristics were then compared with the standard description of "Bergey's Manual of Determinative Bacteriology", 8th edition (Buchanan and Gibbons, 1974).

\subsection{Antibiotic susceptibility testing}

Antibiotics susceptibility test was performed by Kirby-Bauer disc diffusion method (Hudzicki, 2009). Bacterial suspensions were prepared from fresh culture grown overnight onto nutrient agar plates by using sterile normal saline and the turbidity of the suspension was adjusted to $0.5 \mathrm{McFarland}$ Standard that corresponds to 
approximately $1 \times 10^{8} \mathrm{CFU} / \mathrm{mL}$ of suspension. A sterile cotton swab was dipped into the inoculum then streaked on the Mueller-Hinton agar plate properly. Then antibiotic discs impregnated with selected antibiotic discs for each isolate were dispensed onto the dried agar surface using a sterile forceps. The plates were incubated overnight at $37^{\circ} \mathrm{C}$. After incubation period, the resulted zone of inhibition was compared with that of Clinical and Laboratory Standard Institute guideline (CLSI, 2018) for the interpretation of the data and categorization of the test strains as intermediate, sensitive, or resistant (Uddin et al., 2017)

\section{Results and Discussion}

We have retrospectively collected data of 118 patients with invasive bacterial diseases (IBD) of different age groups (0-85 years) (Figure 1) whose 54\% (64) are male and 46\% (64) are female (Figure 2). Among them $26.5 \%$ (31) are 5 or below 5 years of age, $14.53 \%$ (17) are 6-10 years of age. More than half (58\%) of the patient enrolled in this study are of less than 21 years of age.

We found that $25 \%$ (30) blood cultures of enrolled patient (118) in the study is positive growth with any organisms. S. aureus (38\%) and Staphylococcus sp. (38\%) are found as more dominant organisms. S. aureus are involved in diverse range of clinical complications including asymptomatic colonization, skin and soft tissue infection and bone and joint infections. S. aureus is major pathogen of community-acquired pneumonia in children <5 years of age (Schwartz and Nourse, 2012). Staphylococcus sp. is considered as contamination from the skin which indicates aseptic techniques were not maintained properly during collecting blood. S. aureus is an opportunistic pathogen in blood as it is normal flora in the skin. $S$. aureus showed sensitivity to amphicillin $(25 \mu \mathrm{g})$, netilmicin $(30 \mu \mathrm{g})$, ciprofloxacin $(5 \mu \mathrm{g})$, amikacin $(30 \mu \mathrm{g})$, gentamicin $(10 \mu \mathrm{g})$, cloramphenicol $(30 \mu \mathrm{g})$, cotrimoxazole $(25 \mu \mathrm{g})$, ceftriaxone $(30 \mu \mathrm{g})$, azithromicin $(15 \mu \mathrm{g})$, ceftazidime $(30 \mu \mathrm{g})$, clindamicin $(2 \mu \mathrm{g})$ and penicillin (10 unit) (Table 1). As we found $S$. aureus was sensitive to all antibiotics used in this study including gentamicin and ciprofloxacin which are in agreement with other reports from Bangladesh (Ashrafudoulla et al., 2017) and other part of the world (Olayinka et al., 2010). In the USA in 2005, an estimated 94000 invasive MRSA infections required hospitalization and were associated with 19000 deaths (Klevens et al., 2007). However, emergence of multidrug resistant S. aureus has been reported from different parts of the world (Kumar, 2016; Adebola and Tarilate, 2011) as well as from Bangladesh (Ashrafudoulla et al., 2017).

In this study, $17 \%$ (3) of all etiologic agents causing invasive bacterial diseases (IBD) is Salmonella sp. The other etiologic agents of invasive bacterial diseases (IBD) are E. coli (7\%), S. Paratyphii (3\%), Shigella sp. (3\%) and Pseudomonas sp. (3\%) etc. (Figure 3). We found that S. Paratyphii and Salmonella sp. are sensitive to amphicillin $(25 \mu \mathrm{g})$, netilmicin $(30 \mu \mathrm{g})$, amikacin $(30 \mu \mathrm{g})$, gentamicin $(10 \mu \mathrm{g})$, cloramphenicol $(30 \mu \mathrm{g})$, cotrimoxazole $(25 \mu \mathrm{g})$, ceftriaxone $(30 \mu \mathrm{g})$, azithromicin $(15 \mu \mathrm{g})$ and cefixime $(5 \mu \mathrm{g})$ and intermediate sensitive to ciprofloxacin $(5 \mu \mathrm{g})$ which denotes that ciprofloxacin $(5 \mu \mathrm{g})$ is losing its preference to treat patient with invasive disease infected with $S$. Paratyphi and Salmonella sp. (Table 1).

Table 1. Antibiotic susceptibility testing (AST) results of some etiologic agents (Diameter of zone of inhibition in $\mathbf{m m}$ ).

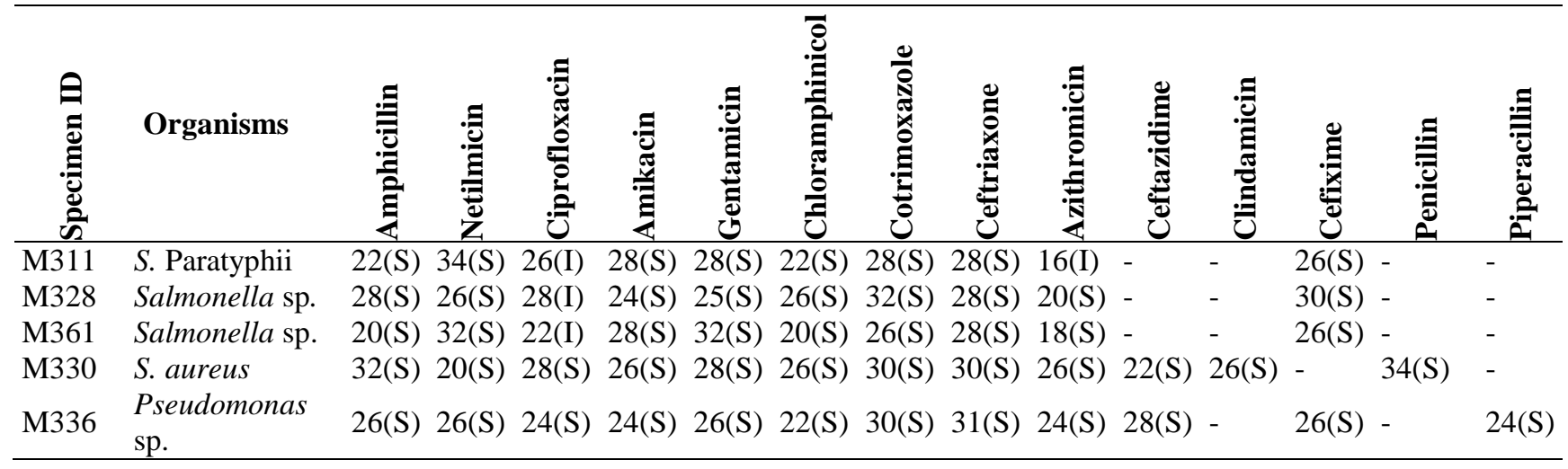

*S-Sensitive; I-Intermediate;

**Results interpreted according CLSI guide line 2018.

Previously in invasive non-typhoidal Salmonella (iNTS) endemic sub sarahan African countries, cotrimoxazole, ampicillin or chloramphenicol were used as first-line treatment for enteric and iNTS diseases. 
However, due to raising prevalence of iNTS which are resistant to commonly used antibiotics, broad spectrum cephalosporins and fluoroquinolones replaced these older agents from the late 1880s. Fluoroquinolone resistance among NTS is an increasing problem. In 1990 ciprofloxacin resistant Salmonella enteric was reported for the first time (Nath et al., 2000). Since then, there have been several reports of ciprofloxacin resistant isolates from different countries including India, Pakistan, Vietnam, Spain and Malawi. (Piddock et al., 1990; Menezes et al., 2010). Multidrug resistant Salmonella Typhi and Salmonella Paratyphi A is a great problem in endemic areas and returning travellers (Wain et al., 2015). In the late 1980s and 1990s, chloramphenicol, ampicillin and co-trimoxazole resistant isolates of Typhi and Paratyphi were involved in large outbreaks in Asia (Arjyal et al., 2011). Ciprofloxacin and Ofloxacin have been broadly recommended for treatment in the last two decades for the patients including children (Effa et al., 2001. Crump et al. (2008) reported the emergences of intermediate level ciprofloxacin resistant isolates which supports our study. This is may be due to extensive use of ciprofloxacin. High-level fluoroquinolone resistance isolates are now commonly found in the Indian sub-continent but are also emerging in Africa (Gaind et al., 2006; Feasey et al., 2014; Koirala et al., 2013).

Pseudomonas sp. showed sensitivity to amphicillin $(25 \mu \mathrm{g})$, netilmicin $(30 \mu \mathrm{g})$, ciprofloxacin $(5 \mu \mathrm{g})$, amikacin $(30 \mu \mathrm{g})$, gentamicin $(10 \mu \mathrm{g})$, cloramphenicol $(30 \mu \mathrm{g})$, cotrimoxazole $(25 \mu \mathrm{g})$, ceftriaxone $(30 \mu \mathrm{g})$, azithromicin $(15 \mu \mathrm{g})$, ceftazidime $(30 \mu \mathrm{g})$, cefixime $(5 \mu \mathrm{g})$ and piperacillin $(100 \mu \mathrm{g})$ (Table 1). Emergence of multidrug resistant Pseudomonas has attracted the attention of many researchers in recent decades (Gomez et al., 2012). According to a recent report by The European Antimicrobial Resistance Surveillance Network (EARS-Net), among $P$. aeruginosa invasive isolates, the mean resistance percentages for piperacillin/tazobactam, carbapenems and fluoroquinolones were close to $20 \%$, while for ceftazidime and aminoglycosides they were 13\% (EARS-Net, 2015). In Europe between 2011 and 2015, carbapenem and ceftazidime resistance trend of $P$. aeruginosa didn't change but raise of piperacillin resistant isolates was observed (EARS-Net, 2015). There were higher resistance rates in the southern and Eastern Europe compared with the northern countries (EARS-Net, 2015). According to a report from Spain, higher resistance rates for piperacillin/tazobactam, ceftazidime, fluoroquinolones and aminoglycosides (with the exception of amikacin) than those reported by EARS-Net (EARS-Net, 2015; Cabot et al., 2011). High Ampicillin resistant isolates were reported from Bangladesh (Nasreen et al., 2015), Pakistan (Anjum and Mir, 2010) and India (Krishnakumar et al., 2012). Isolates which showed high resistance to Gentamicin were also found in Bangladesh (Rashid et al., 2007), Egypt, India (Raja and Singh, 2007), Iran (Saderi et al., 2010), and Pakistan (Naqvi et al., 2005). Previously Co-trimoxazole resistant isolates were found in Bangladesh (Rashid et al., 2007; Nasreen et al., 2015). Rashid et al. (2007) found ciprofloxacin resistant $P$. aeruginosa in Bangladesh. But another study conducted by Nasreen et al. (2015) found no ciprofloxacin resistant isolates, which observation is similar to ours.

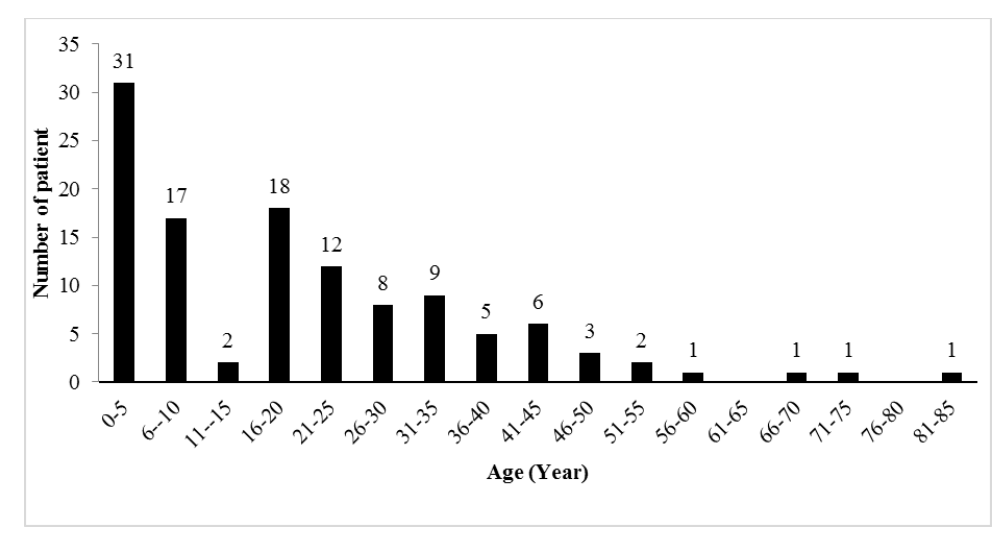

Figure 1. Age distribution of the patients with invasive bacterial diseases (IBD) (Total patient: 117 as we missed age of one patient). 


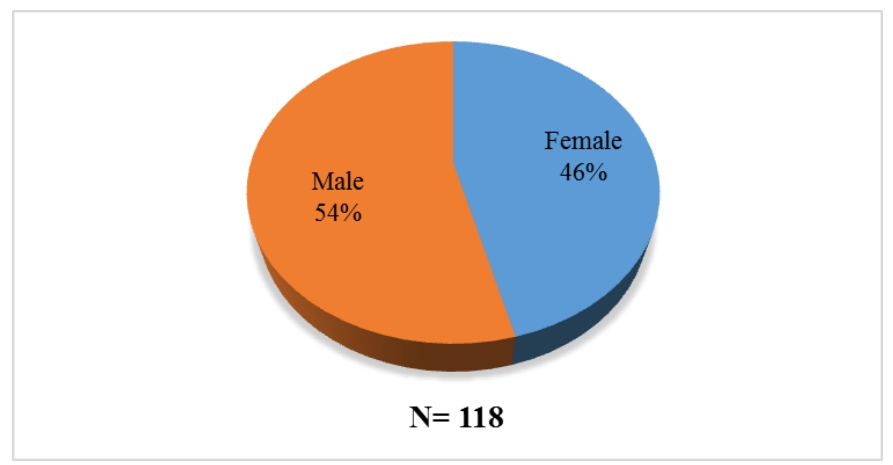

Figure 2. Patients with invasive bacterial diseases (IBD) enrolled in the study were $54 \%$ male and $46 \%$ female.

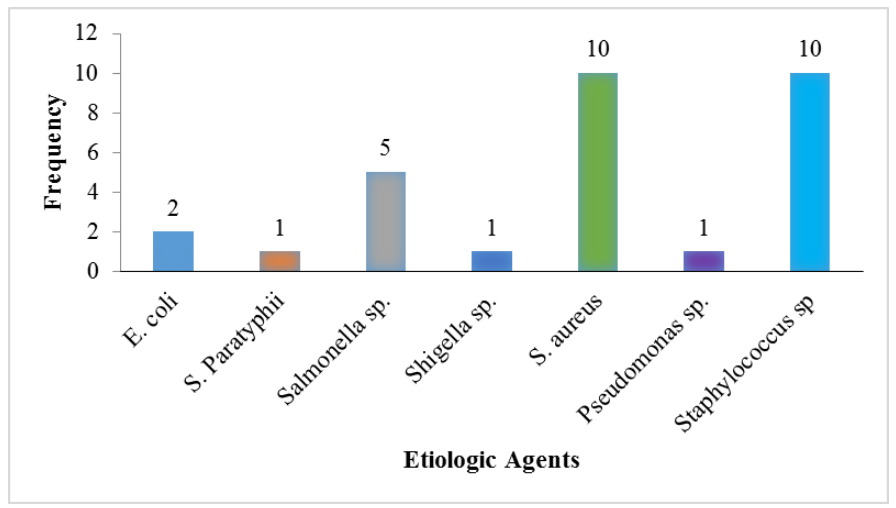

Figure 3. Distribution of etiologic agents causing invasive bacterial diseases (IBD).

\section{Conclusions}

Though this study was not well designed and not adequately powered but it brought some lights in to prevalence of invasive bacterial disease causing by non typhoidal Salmonella (NTS) and S. aureus and their response to commonly used antibiotics. We advised further study needed to be conducted to delineate the entire scenario of invasive bacterial diseases.

\section{Conflict of interest}

None to declare.

\section{References}

Anjum F and A Mir, 2010. Susceptibility pattern of Pseudomonas aeruginosa against various antibiotics. Afr. J. Microbiol. Res., 4: 1005-1012.

Arjyal A, B Basnyat, S Koirala, A Karkey, S Dongol and KK Agrawaal, 2011. Gatifloxacin versus chloramphenicol for uncomplicated enteric fever: An open-label, randomised, controlled trial. Lancet Infect. Dis., 11: 445-454.

Ashrafudoulla M, M Rahman, MFR Mizan, A Begum and SD Ha, 2017. Prevalence of multidrug resistant Staphylococcus aureus in GonoShastho Nagar Hospital, Dhaka, Bangladesh. Afr. J. Microbiol. Res., 11: 1223-1229.

Bryce J, CB Pinto, K Shibuya, RE Black, and the WHO Child Health Epidemiology Reference Group, 2005. WHO estimates of the causes of death in children. Lancet, 365: 1147-52.

Buchanan RE and NE Gibbons, 1974. Bergey's Manual of determinative bacteriology, 8th Edition. Williams and Wilkins Co., Baltimore, pp. 1268.

Cabot G, AA Ocampo-Sosa, F Tubau, MD Macia, C Rodríguez, B Moya, L Zamorano, C Suárez, C Peña, L Martínez-Martínez, A Oliver and Spanish Network for Research in Infectious Diseases (REIPI), 2011. Overexpression of AmpC and efflux pumps in Pseudomonas aeruginosa isolates from bloodstream infections: prevalence and impact on resistance in a Spanish multicenter study. Antimicrob. Agents Chemother., 55: 1906-11. 
Crump JA, K Kretsinger, K Gay, RM Hoekstra, DJ Vugia, S Hurd, SD Segler, M Megginson, LJ Luedeman, B Shiferaw, SS Hanna, KW Joyce, ED Mintz, FJ Angulo and Emerging Infections Program FoodNet and NARMS Working Groups, 2008. Clinical response and outcome of infection with Salmonella enterica Serotype Typhi with decreased susceptibility to fluoroquinolones: a United States FoodNet Multicenter Retrospective Cohort Study. 52: 1278-1284.

Denny FW and FA Loda, 1986. Acute respiratory infections are the leading cause of death in children in developing countries. Am. J. Trop Med. Hyg., 35: 1-2.

Effa E, Z Lassi, J Critchley, P Garner, D Sinclair and PL Olliaro and ZA Bhutta, 2011. Fluoroquinolones for treating typhoid and paratyphoid fever (enteric fever). Cochrane Database Syst. Rev., (10):CD004530.

European Centre for Disease Prevention and Control. Antimicrobial resistance surveillance in Europe 2015, 2017. Annual Report of the European Antimicrobial Resistance Surveillance Network (EARS-Net). Stockholm: ECDC.

Feasey NA, AK Cain, CL Msefula, P Derek, A Maaike, M Aslett, DB Everett, TJ Allain, G Dougan, MA Gordon, RS Heyderman and RA Kingsley, 2014. Drug Resistance in Salmonella enterica ser. Typhimurium Bloodstream Infection, Malawi. Emerg. Infect. Dis., 20: 1957-1959.

Gaind R, B Paglietti, M Murgia, R Dawar, S Uzzau, P Cappuccinelli, M Deb, P Aggarwal and S Rubino, 2006. Molecular characterization of ciprofloxacin-resistant Salmonella enterica serovar Typhi and Paratyphi A causing enteric fever in India. J. Antimicrob. Chemother., 58: 1139-1144.

Gomez MP, J Vega-Baudrit and S Nunez-Corrales, 2012. Overview of multidrug resistant Pseudomonas aeruginosa and novel therapeutic approaches. J. Biomater. Nanobiotechnol., 3: 519-527.

Greenwood BM, 1987. The epidemiology of acute bacterial meningitis in tropical Africa. In: Bacterial meningitis. London, Academic Press, 1987: 61-87.

Guerrant RL, JM Hughes, NL Lima and J Crane, 1990. Diarrhea in Developed and Developing Countries: Magnitude, Special Settings, and Etiologies. Rev. Infect. Dis., 12: S41-S50.

Hossain S, M Rahman, AH Baqui, N Nahar, F Tofaill, VI Mathan and GJ Fuchs, 2000. Hospital-based Surveillance of Invasive Streptococcus pneumoniae and Haemophilus influenzae-associated Diseases in Bangladeshi Children and their Antimicrobial Resistance: A Preliminary Report. Abstract 9th Annual Scientific Conference.

Hudzicki J, 2009. Kirby-bauer disk diffusion susceptibility test protocol.

Kariuki S and G Dougan, 2014. Antibacterial resistance in sub-Saharan Africa: an underestimated emergency. Ann. N. Y. Acad. Sci., 1323: 43-55.

Klein EY, TPV Boeckel, EM Martinez, S Pant, S Gandra, SA Levin, H Goossens and R Laxminarayan, 2018. Global increase and geographic convergence in antibiotic consumption between 2000 and 2015. PNAS, 115: E3463-E3470.

Klevens RM, JR Edwards, CL Richards, TC Horan, RP Gaynes, DA Pollock and DM Cardo, 2007. Estimating Health Care-Associated Infections and Deaths in U.S. Hospitals, 2002. Public Health Reports, 122: 160-166.

Koirala S, B Basnyat, A Arjyal, O Shilpakar, K Shrestha, R Shrestha, UM Shrestha, K Agrawal, KD Koirala, SD Thapa, A Karkey, S Dongol, A Giri, M Shakya, KR Pathak, J Campbell, S Baker, J Farrar, M Wolbers and C Dolecek, 2013. Gatifloxacin versus ofloxacin for the treatment of uncomplicated enteric fever in Nepal: an open-label, randomized, controlled trial. PLoS Negl Trop. Dis., 7: e2523.

Krishnakumar S, RA Rajan, MM Babu and VDM Bai, 2012. Antimicrobial susceptibility pattern of extended spectrum of beta lactamase (ESBL) producing uropathogens from pregnant women. Indian J. Med. and Healthcare, 1: 188-192.

Kumar M, 2016. Multidrug-resistant Staphylococcus aureus, India, 2013-2015. Emerg. Infect. Dis., 22: 16661667.

Lee JH, EK Song, JA Lee, NH Kim, DH Kim, KW Park et al., 2005. Clinical entities and etiology of invasive bacterial infections in apparently healthy children. Korean J. Pediatr., 48: 1193-200.

Liu Y, MA Lee, EE Ooi, Y Mavis, AL Tan and HH Quek, 2003. Molecular typing of Salmonella enterica serovar Typhi isolates from various countries in Asia by a multiplex PCR assay on variable-number tandem repeats. J. Clin. Microbiol., 41: 4388-4394.

Menezes GA, MA Khan, BN Harish, SC Parija, W Goessens, K Vidyalakshmi, S Baliga and JP Hays, 2010. Molecular characterization of antimicrobial resistance in non-typhoidal salmonellae associated with systemic manifestations from India. J. Med. Microbiol., 59: 1477-1483.

Mullholland K, 1999. Strategies for the control of pneumococcal diseases. Vaccine, 1001: S79-84.

Murray CJL and AD Lopez, 1996. Global health statistics; A compendium of incidence, prevalence, and mortality estimates for over 200 conditions. World Health Organization. 
Nam SG and HJ Lee, 1998. Etiology of invasive bacterial infections in apparently healthy children. Korean J, Infect. Dis., 30: 227-234.

Naqvi ZA, K Hashmi, Q Rizwan and SA Kharal, 2005. Multi drug resistant Pseudomonas aeruginosa: a nosocomial infection threat in burn patients. Pakistan J. Pharmacol., 22: 9-15.

Nasreen M, A Sarker, M A Malek, M Ansaruzzaman and M Rahman, 2015. Prevalence and resistance pattern of Pseudomonas aeruginosa isolated from surface water. Advances in Microbiol., 5: 74-81.

Nath G, A Tikoo, H Manocha, AK Tripathi and A Gulati, 2000. Drug resistance in Salmonella typhi in North India with special reference to ciprofloxacin. J Antimicrob. Chemother., 46: 149-150.

Olayinka BO, OS Olonitola, AT Olayinka and B Raji, 2010. Antibiotic susceptibility pattern and multiple antibiotic resistance index of Staphylococcus aureus isolates in Zaria. Nigeria J. Trop. Biosci., 4: 51-54.

Onanuga A and TC Temedie, 2011. Multidrug-resistant intestinal Staphylococcus aureus among self-medicated healthy adults in Amassoma, South-South, Nigeria. J. Health Popul. Nutr., 29: 446.

Piddock LJ, C Wray, I McClaren and R Wise, 1990. Quinolone resistance in Salmonella spp: veterinary pointers. Lancet, 336: 125.

Raja NS and NN Singh, 2007. Antimicrobial Susceptibility Pattern of Clinical Isolates of Pseudomonas aeruginosa in a Tertiary Care Hospital. J. Microbiol. Immunol.and Infec., 40: 45-49.

Rashid A, A Chowdhury, SHZ Rahman, SA Begum and N Muazzam, 2007. Infections by Pseudomonas aeruginosa and Antibiotic Resistance Pattern of the Isolates from Dhaka Medical College Hospital. Bangladesh J. Med. Microbiol., 1: 48-51.

Saderi H, H Lotfalipour, P Owlia and H Salimi, 2010. Detection of metallo- $\beta$-lactamase producing Pseudomonas aeruginosa isolated from burn patients in Tehran, Iran. Laboratory Medicine, 41: 609-612.

Schuchat A, T Hilger, E Zell, MM Farley, A Reingold, L Harrison, L Lefkowitz, R Danila, K Stefonek, N Barrett, D Morse, R Pinner and Active Bacterial Core Surveillance Team of the Emerging Infections Program Network, 2001. Active bacterial core surveillance of the emerging infections program network. Emerg. Infect. Dis., 7: 92-99.

Schwartz KL and C Nourse, 2012. Panton-Valentine leukocidin-associated Staphylococcus aureus necrotizing pneumonia in infants: a report of four cases and review of the literature. Eur. J. Pediatr., 171: 711-717.

Shann F, 1986. Etiology of severe pneumonia in children in developing countries. Paediatr. Infect. Dis., 5: 24752.

Shann F, 1995. The management of pneumonia in children in developing countries. Clin. Infect. Dis., 21: S218225.

Uddin, MS, MI Hoq, MS Ali, MM Rahman and KMS Islam, 2017. Antibiotic resistance pattern of Salmonella spp. isolated from stool samples of hospitalized diarrheal patients in Bangladesh. Asian J. Med. Biol. Res., 3: 534-538.

UNICEF/WHO, 2006. Pneumonia: The forgotten killer of children.

Wain J, RS Hendriksen, ML Mikoleit, KH Keddy and RL Ochiai, 2015. Typhoid fever. Lancet, 385: 1136-1145. 